\title{
PARP-1 may be involved in hydroquinone-induced apoptosis by poly ADP-ribosylation of ZO-2
}

\author{
JIAXIAN LIU ${ }^{1}$, QIAN YUAN ${ }^{1}$, XIAOXUAN LING ${ }^{1,2}$, QIANG TAN $^{3}$, HAIRONG LIANG $^{1}$, JIALONG CHEN $^{4}$,

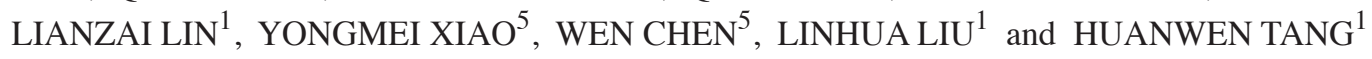 \\ ${ }^{1}$ Department of Environmental and Occupational Health, Dongguan Key Laboratory of Environmental Medicine, \\ School of Public Health, Guangdong Medical University, Dongguan, Guangdong 523808; ${ }^{2}$ The State Key Laboratory \\ of Respiratory Disease, The Institute for Chemical Carcinogenesis, Collaborative Innovation Center for \\ Environmental Toxicity, School of Public Health, Guangzhou Medical University, Guangzhou, Guangdong 511436; \\ ${ }^{3}$ General Office, Foshan Institute of Occupational Disease Prevention and Control, Foshan, Guangdong 528000; \\ ${ }^{4}$ Department of Occupational Health and Occupational Medicine, Guangdong Provincial Key Laboratory of Tropical \\ Disease Research, School of Public Health and Tropical Medicine, Southern Medical University, Guangzhou, \\ Guangdong 510515; ${ }^{5}$ Department of Toxicology, Guangzhou Key Laboratory of Environmental Pollution and Health \\ Risk Assessment, School of Public Health, Sun Yat-sen University, Guangzhou, Guangdong 510080, P.R. China
}

Received August 25, 2016; Accepted May 17, 2017

DOI: $10.3892 / \mathrm{mmr} .2017 .7643$

\begin{abstract}
Hydroquinone (HQ), a major reactive metabolite of benzene, contributes to benzene-induced leukemia. The molecular mechanisms that underlie this activity remain to be elucidated. Poly ADP-ribosylation (PARylation) is a type of reversible posttranslational modification that is performed by enzymes in the PAR polymerase (PARP) family and mediates different biological processes, including apoptosis. Zona occludens 2 (ZO-2) is a tight junction scaffold protein, which is involved in cell proliferation and apoptosis. The present study investigated the activity and mechanisms regulated by PARP-1 during HQ-induced apoptosis using TK6 lymphoblastoid cells and PARP-1-silenced TK6 cells. The results revealed that exposure to $10 \mu \mathrm{M}$ HQ for $72 \mathrm{~h}$ induced apoptosis in TK6 cells and that apoptosis was attenuated in PARP-1-silenced TK6 cells. In cells treated with HQ, inhibition of PARP-1 increased the expression of
\end{abstract}

Correspondence to: Professor Huanwen Tang or Dr Linhua Liu, Department of Environmental and Occupational Health, Dongguan Key Laboratory of Environmental Medicine, School of Public Health, Guangdong Medical University, 1 Xincheng Road, Dongguan, Guangdong 523808, P.R. China

E-mail: thw@gdmu.edu.cn

E-mail: linhua-liu@163.com

Abbreviations: HQ, hydroquinone; PARP-1, poly(ADP-ribose) polymerase-1; PARylation, poly ADP-ribosylation; PAR, poly (ADP-ribose); $\mathrm{NAD}^{+}$, donor nicotinamide adenine dinucleotide; ZO-2, zona occludens 2; ROS, reactive oxygen species

Key words: hydroquinone, ZO-2, PARP-1, poly ADP-ribosylation, apoptosis, lymphoblastoid cells
B cell leukemia/lymphoma 2 (Bcl-2), increased ATP production and reduced reactive oxygen species (ROS) production relative to the levels observed in cells treated with HQ alone. Co-localization of ZO-2 and PAR (or PARP-1 protein) was determined using immunofluorescence confocal microscopy. The findings of the present study revealed that ZO-2 was PARylated via an interaction with PARP-1, which was consistent with an analysis of protein expression that was performed using western blot analysis, which determined that ZO-2 protein expression was upregulated in HQ-treated control cells and downregulated in HQ-treated PARP-1-silenced TK6 cells. These findings indicated that prolonged exposure to a low dose of HQ induced TK6 cells to undergo apoptosis, whereas inhibiting PARP-1 attenuates cellular apoptosis by activating Bcl-2 and energy-saving processes and reducing ROS. The present study determined that PARP-1 was involved in HQ-induced apoptosis by PARylation of ZO-2.

\section{Introduction}

Apoptosis is a spontaneous cell death subroutine, that is triggered by various physiological and pathological stimuli and that has distinct morphological characteristics and energy-dependent mechanisms. Apoptosis has a crucial role in a variety of processes, including normal cell population maintenance, the appropriate development of the immune system, embryonic development and chemical-induced cell death. Inappropriate apoptosis (either too little or too much) may lead to neurodegenerative diseases, autoimmune disorders, ischemic damage and various types of cancer (1). Previous studies have made substantial progress in the biochemical and genetic exploration of apoptosis, resulting in the identification of many key apoptotic genes, signaling pathways and specific biochemical features (2), the molecular mechanisms that underlie apoptosis remain to be fully elucidated. 
Apoptosis is believed to be strictly regulated by various key apoptotic genes, including those in the caspase family, the B cell leukemia/lymphoma 2 (Bcl-2) family, the poly(ADP-ribose) polymerase (PARP) family, oncogenes and tumor suppressor genes. Poly ADP-ribosylation (PARylation), a type of reversible protein modification, is performed by the enzymes in the PARP family and poly(ADP-ribose) (PAR)-degrading enzymes, such as PAR glycohydrolase (3), ADP-ribosyl hydrolase 3 (4) and members of the nucleoside diphosphate linked moiety $\mathrm{X}$ family of proteins (5). It has been previously estimated that $\sim 90 \%$ of PAR polymers are formed via the catalysis of PARP-1, which was positively correlated with PARP activity. PARP-1 is activated primarily by DNA breaks; however, it may also be activated by alternative mechanisms, such as phosphorylation. Activated PARP-1 and PARylation regulate various cellular machineries, including those involved in cell death (6). A previous study suggested that PARylation and PARP-1 mediate several cell death subroutines, including necrosis (necroptosis), parthanatos, autophagy and apoptosis (7). Previous studies have demonstrated that inhibiting PARP activity or knocking out/silencing PARP-1 may sensitize cells to the cytotoxic effects of ionizing radiation or DNA-damaging agents, such as DNA alkylates, cisplatin and topoisomerase poisons $(8,9)$. However, whether PARylation by PARP-1 affects the apoptotic process remains to be determined. In response to mild DNA damage, PARylation facilitates DNA repair and therefore survival, whereas more severe genotoxic stimuli may activate the apoptotic pathway. Severe DNA damage may lead to in apoptosis or necrosis, which are attributed to the depletion of donor nicotinamide adenine dinucleotide $\left(\mathrm{NAD}^{+}\right) / \mathrm{ATP}$ by the excessive activation of PARP-1 (10).

Hydroquinone (HQ) is a major active metabolite of benzene and is commonly used as a substitute for benzene in in vitro experiments. Benzene is a confirmed human carcinogen that was proposed to be associated with myelodysplastic syndromes, acute myeloid leukemia, non-Hodgkin lymphoma and childhood leukemia $(11,12)$. However, previous in vivo and in vitro studies have determined that the pathways through which HQ contributes to benzene-induced leukemia may also include oxidative stress, DNA damage, cell cycle regulation and apoptosis (13-15), the underlying molecular mechanisms involved in HQ toxicity remain unclear. Our previous study revealed that HQ leads to abnormal cell cycle progression that is accompanied with the upregulation of PARP-1 in TK6 cells (16). A previous study, based on the specificity of the synthesized substrate $\mathrm{NAD}^{+}$used affinity purification and tandem mass spectrometry and revealed that zona occludens 2 (ZO-2) is a target of PARP-1 in HEK 293T nuclear lysates (17). ZO-2, a 160-kDa protein (18), belongs to the family of membrane-associated guanylate kinase (MAGUK) homologs, which localize on the cytoplasmic surface of intercellular contacts in epithelial and endothelial cells and have also been observed to be distributed in the nucleus. ZO-2 affects various cellular processes, including cell proliferation, apoptosis, stress tolerance and barrier integrity $(19,20)$. It is of note that the importance of ZO-2 in lymphoblastoid cells remains to be determined. The present study used TK6 lymphoblastoid cells and PARP-1-silenced TK6 cells to investigate cell death and cell fate specification following prolonged exposure to
HQ. The present study also aimed to determine the role of pleiotropic PARP-1 in these processes and to identify the association between PARP-1 and ZO-2.

\section{Materials and methods}

Chemicals and reagents. HQ, DAPI and mouse anti-human PAR (cat. no. MAB3192) primary antibody were purchased from Sigma-Aldrich (Merck KGaA, Darmstadt, Germany). Rabbit anti-human Bcl-2 (cat. no. ab32124), caspase-3 (cat. no. ab408) primary antibodies and a 2',7'-dichlorofluorescin diacetate (DCFDA)-Cellular Reactive Oxygen Species Detection assay kit were purchased from Abcam (Cambridge, MA, USA). Rabbit anti-human PARP-1 antibody (cat. no. CST 9542), ZO-2 (cat. no. CST 2847), Bax (cat. no. CST 5023P), GAPDH (cat. no. CST 2118), mouse anti-human $\alpha$-tubulin (cat. no. CST 3873) primary antibodies, and Alexa Fluor 555-conjugated (CST 4409s) and 488-conjugated (cat. no. CST 4412s) secondary antibodies were acquired from Cell Signaling Technology (Danvers, MA, USA). Mouse anti-human PARP-1 antibodies (cat. no. sc8007) and horseradish peroxidase-conjugated goat anti-rabbit (cat. no. sc2004) or goat anti-mouse IgG (cat. no. sc2005) antibodies were obtained from Santa Cruz Biotechnology (Dallas, TX, USA). Primers, TRIzol, ECL-PLUS chemiluminescence assay and RevertAid First Strand cDNA Synthesis kits were obtained from Thermo Fisher Scientific, Inc. (Waltham, MA, USA). CellTiter-Glo Luminescent Cell Viability and Caspase-Glo 3/7 assay kits were obtained from Promega Corporation (Madison, WI, USA). FastStart Universal SYBR Green Master mix was obtained from Roche Diagnostics (Mannheim, Germany). Annexin V-FITC Apoptosis Detection kit was obtained from Keygen Biotech, Co., Ltd. (Nanjing, China).

Cell cultures and chemical treatments. The TK6 lymphoblastoid cell line was provided by Professor Lishi Zhang (Sichuan University, Chengdu, China). The cell properties, culture conditions and chemical treatment methods used in the present study have been described in our previous study (21). The PARP-1-silenced TK6 cells, which stably express PARP-1-short hairpin (sh)RNA have been termed as shPARP-1, were provided by Professor Tang (Guangdong Medical University, Dongguan, China) (22). Empty TK6 cells were used as control cells. The experimental cells were treated with $10 \mu \mathrm{M}$ HQ for $72 \mathrm{~h}$, and the control cells were treated with PBS.

Western blot analysis. Total proteins were extracted from prepared cells using cell lysis buffer (Cell Signaling Technology, Inc.), and their concentrations were determined using bicinchoninic acid (BCA) assays (Beijing ComWin Biotech Co., Ltd., Beijing, China). Equal quantities $(25 \mu \mathrm{g})$ of protein were separated using SDS-PAGE on a $10 \%$ gel and then transferred to PVDF membranes. The membranes were blocked with $5 \%$ fat free milk for $50 \mathrm{~min}$ at room temperature. The target proteins and the loading control proteins were quantified on the same membrane by dividing it into pieces according to the known molecular weights of pre-stained protein standards. The membranes were incubated at $4^{\circ} \mathrm{C}$ overnight with primary antibodies against ZO-2 (1:2,000), 
PARP-1 (1:5,000), Bcl-2 (1:2,000), caspase-3 $(1: 2,000)$ or Bax $(1: 3,000)$. They were then incubated at room temperature for $1 \mathrm{~h}$ with the corresponding species-specific secondary antibodies. GAPDH $(1: 5,000)$ or $\alpha$-tubulin $(1: 10,000)$ were used as internal control. The immunoreactivity of the bands on the membranes was visualized using ECL-PLUS chemiluminescence reactions. Three repeats of the densitometric analysis were performed using Quantity One software, version 4.6.2 (Bio-Rad Laboratories, Inc., Hercules, CA, USA).

Reverse transcription-quantitative polymerase chain reaction $(R T-q P C R)$. Total RNA was isolated, analyzed and transcribed into cDNA as previously described (16). PCR reactions were performed using FastStart Universal SYBR Green Master on a PikoReal 96 Real-Time PCR system (Thermo Fisher Scientific, Inc.). The following forward (F) and reverse (R) primers were used: ZO-2-F, 5'-CCGTTGCTGGTAATGAAACTCCT-3' and ZO-2-R, 5'-TTATCTTGCTCCTCACTGCTCTCTC-3'. The qPCR conditions were as follows: Initial cycle was $95^{\circ} \mathrm{C}$ for $10 \mathrm{~min}$, followed by 40 cycles at $95^{\circ} \mathrm{C}$ for $15 \mathrm{sec}$ and $58^{\circ} \mathrm{C}$ for $40 \mathrm{sec}$. Dissociation curves were used to confirm specificity. Expression levels were calculated using the $2^{-\Delta \Delta C q}$ method (23) with GAPDH as selected internal control.

Flow cytometry. The collected cells were suspended in binding buffer and then stained with Annexin V-FITC Apoptosis Detection kit and propidium iodide according to the manufacturer's protocol. The cells were incubated at room temperature for $15 \mathrm{~min}$ in the dark and then immediately analyzed using a BD FACSCanto II flow cytometer (excitation $=488 \mathrm{~nm}$; emission=530 nm) (BD Biosciences, San Jose, CA, USA). A minimum of 10,000 cells per sample were acquired and analyzed using FlowJo software, version 7.6.1 (Tree Star, Inc., Ashland, OR, USA).

Quantification of caspase-3/7 activity and ATP production. Cell numbers were counted using Countstar IC1000 (Ruiyu Biotech Co., Ltd, Shanghai, China). Caspase-3/7 activity and ATP levels were quantified using Caspase-Glo 3/7 assay kits and CellTiter-Glo Luminescent Cell Viability assays according to the manufacturer's protocol in each kit. For the Caspase-3/7 assays, $100 \mu \mathrm{l}$ of suspended cells were plated in 96-well plates and $100 \mu \mathrm{l}$ Caspase-Glo 3/7 reagent was added. The mixture was agitated for $2 \mathrm{~min}$ and then incubated for $10 \mathrm{~min}$ at room temperature. For the ATP assays, $100 \mu 1$ suspended cells were plated in 96-well plates and $100 \mu \mathrm{l}$ CellTiter-Glo reagent was then added. The mixtures were agitated for $2 \mathrm{~min}$ and then incubated for $5 \mathrm{~min}$ at room temperature. The generated luminescent signal was read using the luminescent detection method on a BioTek Synergy2 microplate reader (BioTek Instruments, Inc., Winooski, VT, USA). The luminescent signal readout was normalized according to the respective cell number and was recorded as the caspase-3/7 or ATP luminescence value.

Indirect immunofluorescence staining. A previously described protocol used to prepare cells for staining (16). Cells were sequentially incubated with either anti-PARP-1 and ZO-2 primary antibodies or anti-PAR and ZO-2 primary antibodies at a dilution of 1:500 at room temperature for $1 \mathrm{~h}$ and then Alexa Fluor 555-conjugated and Alexa Fluor 488-conjugated secondary antibodies at a dilution of 1:1,000 at room temperature for $30 \mathrm{~min}$. Cell nuclei were counterstained using DAPI. The cells were smeared on coverslips and mounted in $90 \%$ glycerol in PBS. They were subsequently imaged using an Olympus FLUOVIEW FV1000 confocal laser-scanning microscope (Olympus Corporation, Tokyo, Japan).

Quantification of ROS production. Reactive oxygen species (ROS) production was determined using a DCFDA-Cellular Reactive Oxygen Species Detection assay kit according to the manufacturer's protocol. Cells $\left(5 \times 10^{5}\right)$ were treated with $10 \mu \mathrm{M}$ HQ for $72 \mathrm{~h}$. The collected cells were then washed in PBS and stained with $20 \mu \mathrm{M}$ DCFDA in $1 \mathrm{X}$ buffer for $30 \mathrm{~min}$ at $37^{\circ} \mathrm{C}$ in the dark. The stained cells were then washed, resuspended in $400 \mu 11 \mathrm{X}$ buffer, and equally seeded in four wells of an opaque 96-well microplate. Staining intensities were immediately determined using a BioTek Synergy2 microplate reader set for an excitation wavelength of $485 \mathrm{~nm}$ and an emission wavelength of $535 \mathrm{~nm}$.

Statistical analysis. Data are presented as the mean \pm standard deviation of at least three independent experiments. One-way analysis of variance was used for comparisons of means among multiple groups, followed by the Student-Newman-Keuls post hoc test. $\mathrm{P}<0.05$ was considered to indicate a statistically significant difference.

\section{Results}

Expression of PARP-1 in PARP-1-silencing TK6 cells. PARP-1 is a pleiotropic molecule that has an important role in various cell death processes. PARP-1-silenced TK6 cells were generated to elucidate the underlying mechanisms involving PARP-1. In these cells, the PARP-1 protein expression was reduced by $75 \%$, indicating that PARP-1 was efficiently silenced $(\mathrm{P}<0.05$; Fig. 1$)$.

Inhibiting PARP-1 attenuates HQ-induced apoptosis. In order to determine the level of apoptosis induced by HQ treatment and the role of PARP-1 in this process, control cells and shPARP-1 cells were treated with PBS or $10 \mu \mathrm{M} \mathrm{HQ}$, respectively, for $72 \mathrm{~h}$ and then subjected to flow cytometry analysis. A higher number of apoptotic cells was observed in the HQ-treated control cells than in the PBS group. The number of apoptotic cells was lower in the PBS-treated shPARP-1 cells compared with the PBS-treated control cells. Additionally, the HQ-treated shPARP-1 cells also had a reduced number of apoptotic cells compared with the HQ-treated control cells, indicating that prolonged exposure to low levels of HQ induced apoptosis and that this effect was attenuated by PARP-1 knockdown (Fig. 2A and B). Changes in the apoptotic rate may lead to altered cell numbers; therefore, cell counting was performed. It was determined that the number of cells in the HQ-treated control cell group was lower when compared with the number of cells in the control + PBS group. The number of cells in the shPARP-1 group was higher compared with the control cells + HQ group (Fig. 2C). These findings suggested that HQ mediated growth inhibition by inducing apoptosis in TK6 cells.

To investigate the underlying mechanism of HQ-induced apoptosis, $\mathrm{Bcl}-2$, caspase-3 and Bax were detected using western 
A

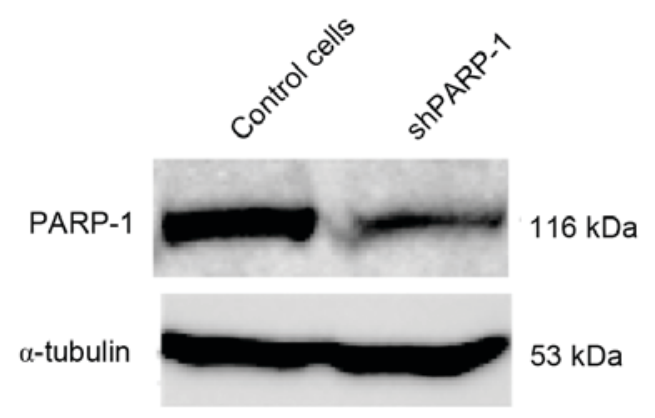

B

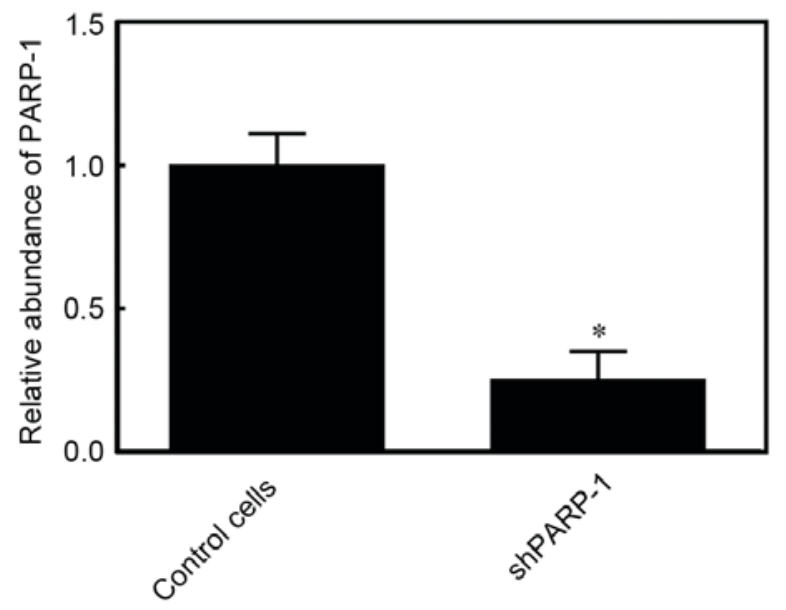

Figure 1. Efficiency of PARP-1 silencing in TK6 cells. (A) Representative images of western blot analysis. Compared to the control cells, PARP-1 was silenced in shPARP-1 cells. (B) Densitometric analysis of PARP-1 protein expression levels. Data are presented as the mean \pm standard deviation of three independent experiments. "P<0.05 vs. control cells. PARP-1, poly (ADP-ribose) polymerase-1; shPARP-1, short hairpin PARP-1.

blot analysis. Bcl-2 was present at lower levels in HQ-treated control cells compared with the PBS-treated cells and at higher levels in the HQ-treated shPARP-1 cells compared with the HQ-treated control cells. Caspase-3 was present at lower levels in the HQ-treated control cells. However, no significant difference in terms of caspase-3 expression was observed between the HQ-treated shPARP-1 cells and the HQ-treated control cells. Bax expression remained constant in both HQ treatment and PARP-1 knockdown (Fig. 2D). These findings suggested that HQ induced the downregulation of Bcl-2 and caspase-3 in TK6 cells. PARP-1 inhibition antagonized the downregulation of Bcl-2 that was induced by HQ treatment.

Caspase-3/7 are key regulators of apoptosis and the activity of these two enzymes indirectly reflects the downstream cascade reaction of cell apoptosis. Consistent with the increase that was observed in the proportion of apoptotic cells in the control cells treated with HQ, an increase in caspase-3/7 activity was also observed in these cells. However, higher caspase-3/7 activity was also observed in shPARP-1 + HQ cells compared with the control cells + HQ and this was inconsistent with the results observed in flow cytometry (Fig. 2E). These findings indicated that inhibition of PARP-1 attenuated HQ-induced apoptosis, this effect may not be achieved via the caspase signaling pathway.

ATP levels were also quantified in order to determine the mechanisms involved in HQ-induced apoptosis as PARP-1 also participates in energy metabolism. More ATP was produced in the control cells treated with HQ; however, the level of ATP was higher in the HQ-treated shPARP-1 cells compared with the HQ-treated control cells (Fig. 2F). These data indicated that the energy-saving effect that was induced by silencing PARP-1 may be involved in protecting against HQ-induced apoptosis. These data also support a previous study that determined that PARP-1 affects ATP depletion (10). Additionally, ROS production was significantly increased in the HQ-treated control cells compared with the PBS only group $(\mathrm{P}<0.05$; Fig. 2G). A considerably reduced level of ROS production was observed in the HQ-treated shPARP-1 cells compared with the HQ-treated control cells (Fig. 2G), indicating that inhibition of
PARP-1 expression may attenuate HQ-induced apoptosis by reducing ROS production.

PARP-1 protein is required for the abundance of the $\mathrm{ZO}-2$ protein. A previous study described a rational design that includes orthogonal $\mathrm{NAD}^{+}$analogue-engineered PARP pairs that may be used to identify the direct protein targets of individual PARPs (17). In this design, ZO-2 was found to be PARylated by PARP-1 (Fig. 3A) (17). Additionally, ZO-2 was reported to be involved in apoptosis (19). The present study aimed to further investigate the association between PARP-1, ZO-2 and apoptosis and detected the ZO-2 and PARP-1 protein expression levels using western blot analysis. PARP-1 was upregulated in the control cells treated with $10 \mu \mathrm{M}$ HQ for $72 \mathrm{~h}$, consistent with the findings of our previous study (21). PARP-1 was effectively inhibited in shPARP-1 cells. PARP-1 levels were lower in shPARP-1 cells treated with HQ compared with HQ-treated control cells; however, shPARP-1 cells treated with HQ expressed higher levels of PARP-1 than shPARP-1 cells treated with PBS (Fig. 3B). The expression levels of ZO-2 changed in parallel to the changes observed in PARP-1 (Fig. 3B). ZO-2 protein expression was upregulated in control cells treated with HQ, whereas in the shPARP-1 cells, where PARP-1 expression was reduced, ZO-2 levels were lower than the levels in the control cells in the group treated with PBS and HQ (Fig. 3B). These findings indicated that PARP-1 and ZO-2 are involved in HQ-induced apoptosis and that the expression of ZO-2 is dependent on PARP-1. To reveal the mechanism by which ZO-2 is dependent on PARP-1, the mRNA expression of ZO-2 was detected using RT-qPCR. It is of note that mRNA levels of ZO-2 were the reverse to those observed for the $\mathrm{ZO}-2$ protein (Fig. 3C), indicating that ZO-2 protein levels were post-translationally regulated by PARP-1.

ZO-2 is modified by PARylation. PARylation, a post-translational modification, is primarily catalyzed by PARP-1. The present study determined whether ZO-2 is modified by PARylation during HQ-induced apoptosis by analyzing the co-localization of ZO-2 and PAR (or PARP-1) using 
A
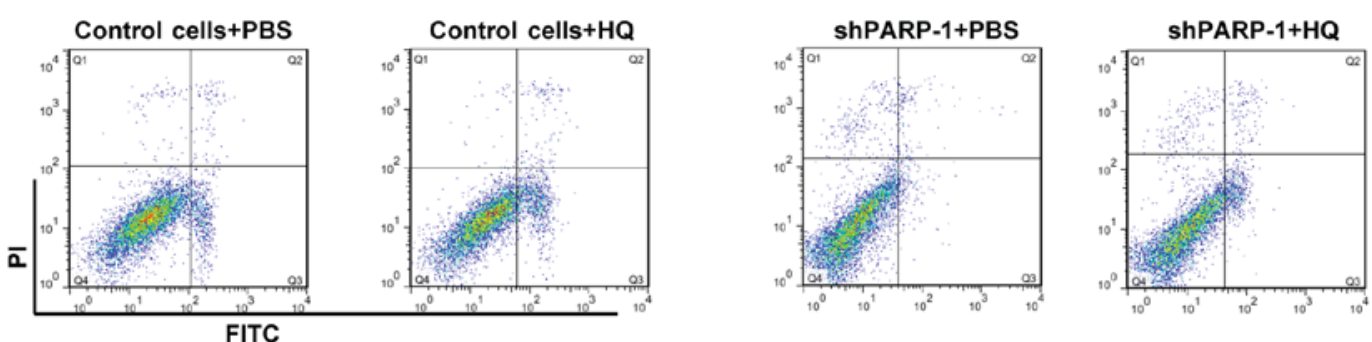

B

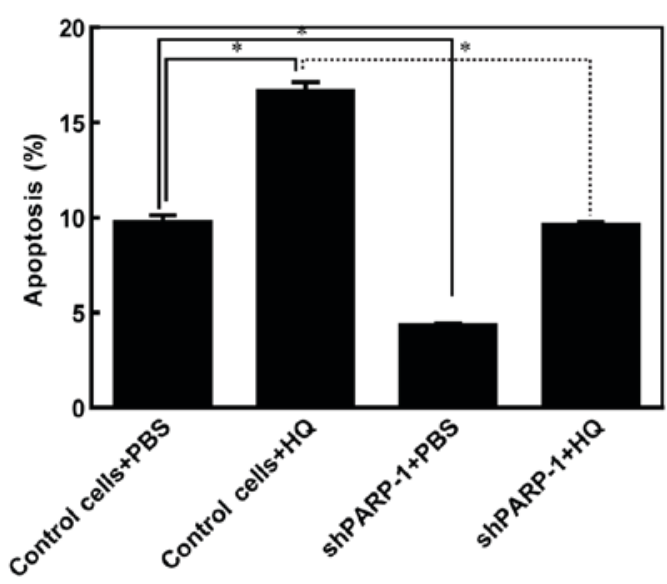

C

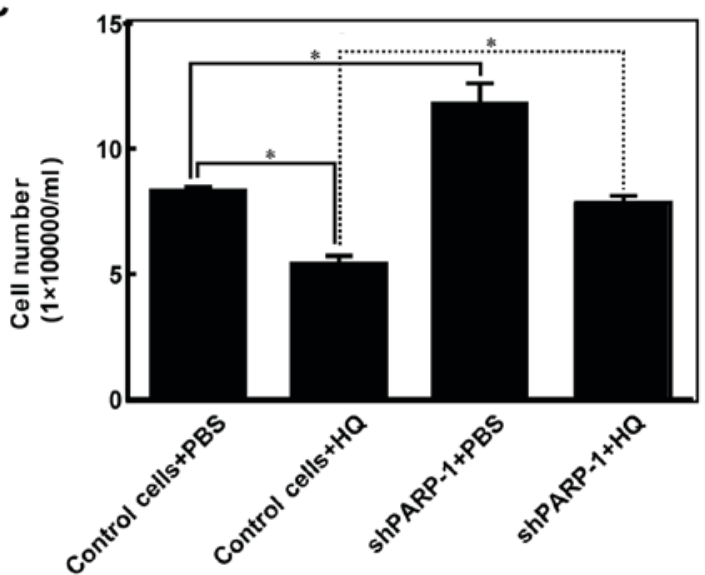

D

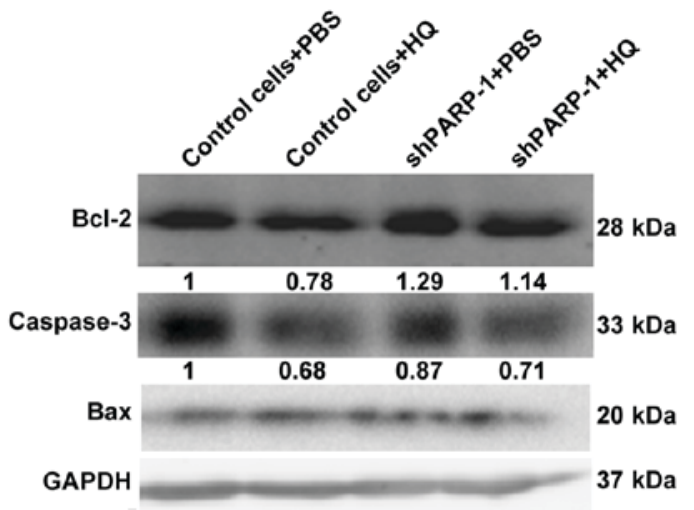

E

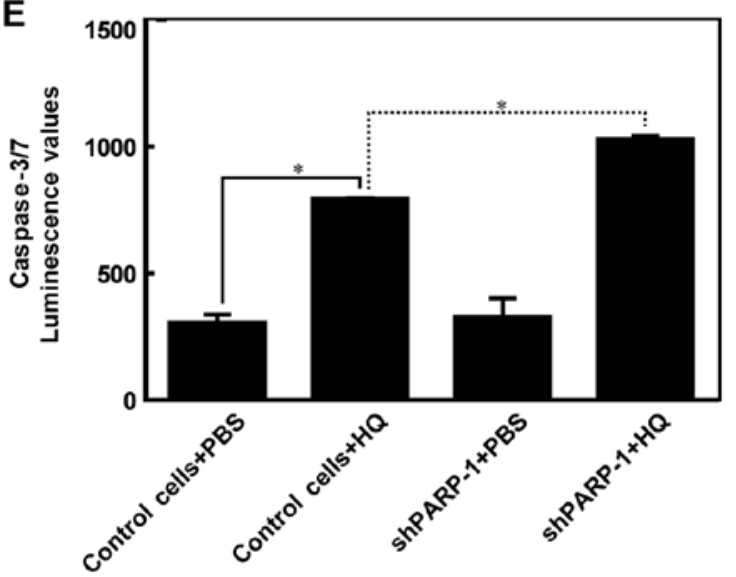

$\mathbf{F}$
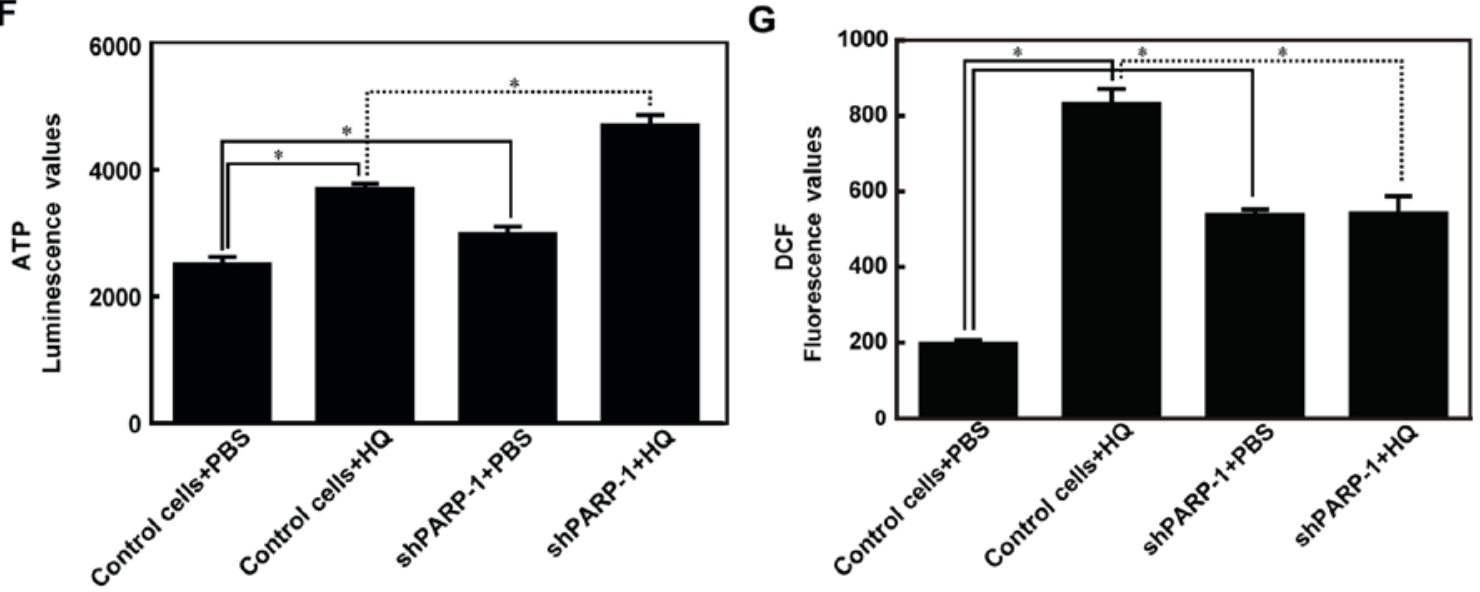

Figure 2. PARP-1 attenuated HQ-induced apoptosis. Empty TK6 cells were used as control cells. Cells were treated with PBS or $10 \mu \mathrm{M}$ HQ for $72 \mathrm{~h}$. (A) Representative images of flow cytometry using Annexin V-FITC and PI double-stained cells. (B) Cell apoptosis rates are presented as the mean \pm standard deviation for at least three independent experiments. (C) Number of cells was counted using Countstar (IC1000). (D) Protein expression levels of the Bcl-2, caspase- 3 and Bax proteins were detected using western blot analysis. (E) Caspase-3/7 luminescence values, which indicate caspase-3/7 activity, were detected using Caspase-Glo-3/7 assay kits. (F) ATP luminescence values, which indicate ATP level, were measured using CellTiter-Glo Luminescent Cell Viability assay kits. (G) Reactive oxygen species production was measured using DCFDA. "P<0.05. HQ, hydroquinone; PARP-1, poly (ADP-ribose) polymerase-1; shPARP-1, short hairpin PARP-1; Bcl2, B cell leukemia/lymphoma 2; Bax, BCL2 associated X; DCFDA, 2',7'-dichlorofluorescin diacetate; FITC, fluorescein isothiocyanate; PI, propidium iodide. 
A

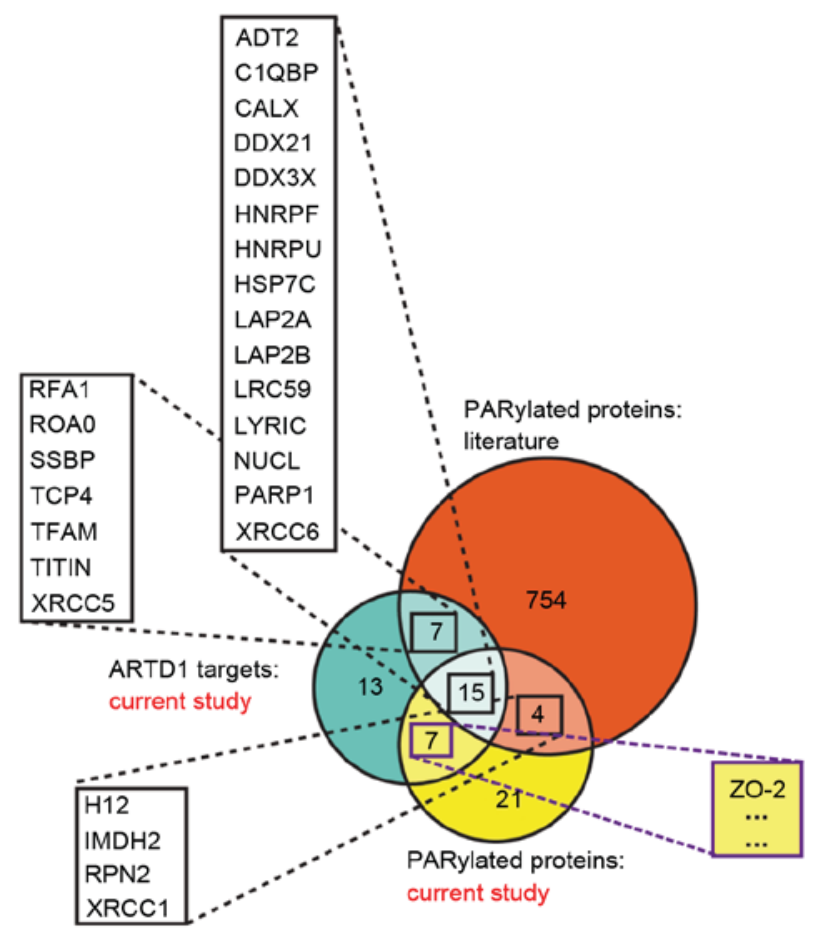

B

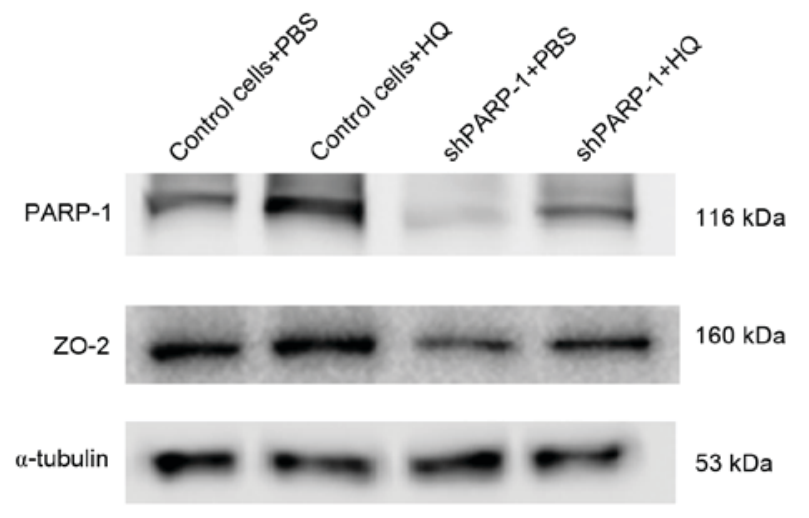

C

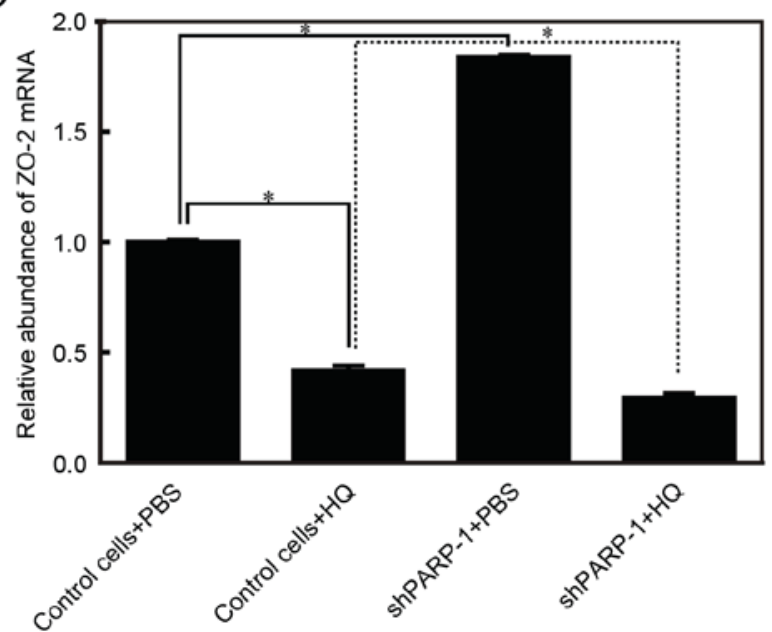

Figure 3. PARP-1 protein is required for the abundance of the ZO-2 protein. (A) Venn diagram showing the direct targets of PARP-1 that were identified by Carter-O'Connell et al (17). (B) PARP-1 and ZO-2 protein expression levels were detected using western blot analysis in control cells and shPARP-1 cells that were treated with $10 \mu \mathrm{M}$ HQ for $72 \mathrm{~h}$. (C) ZO-2 mRNA expression was analyzed and normalized to GAPDH in reverse transcription-quantitative polymerase chain reaction. ${ }^{\mathrm{P}}<0.05$. HQ, hydroquinone; PARP-1, poly (ADP-ribose) polymerase-1; shPARP-1, short hairpin PARP-1; ZO-2, zona occludens 2.

immunofluorescence confocal microscopy. It was revealed that ZO-2 was clearly integrating with PAR (Fig. 4, upper panel) and with PARP-1 respectively (Fig. 4, lower panel), indicating that ZO-2 was PARylated via an interaction with PARP-1. The abundance of ZO-2 in the nucleus was dependent on the abundance of PARP-1 (Fig. 4). These data are consistent with the findings from the western blot analysis.

\section{Discussion}

Previous studies have revealed that different doses of HQ may induce apoptosis $(24,25)$. Treatment with a low dose of $\mathrm{HQ}$ for $24 \mathrm{~h}$ induced HQ-mediated apoptosis in murine fetal livers and bone marrow hematopoietic cells (24). Treatment with a high dose of HQ for $24 \mathrm{~h}$ promoted apoptosis in bone marrow-derived mesenchymal stem cells (25). Treatment with 50 or $100 \mu \mathrm{M}$ HQ for $14 \mathrm{~h}$ promoted apoptosis in W7.2 rfau cells (26). In the present study, treating cells with $10 \mu \mathrm{M}$ $\mathrm{HQ}$ for $72 \mathrm{~h}$ promoted apoptosis. The balance between the pro-apoptotic (Bcl2 associated agonist of cell death and Bax) and anti-apoptotic (Bcl-2 and Bcl-extra large) members of the Bcl-2 family is critical for controlling mitochondria-induced apoptosis (27). Activated caspases may also act as biochemical markers of apoptotic cascade reactions. The present study, consistent with the findings of the flow cytometry experiments, determined that caspase-3/7 activity was activated, caspase- 3 protein levels were upregulated and ATP production was upregulated in TK6 cells treated with HQ, indicating that 

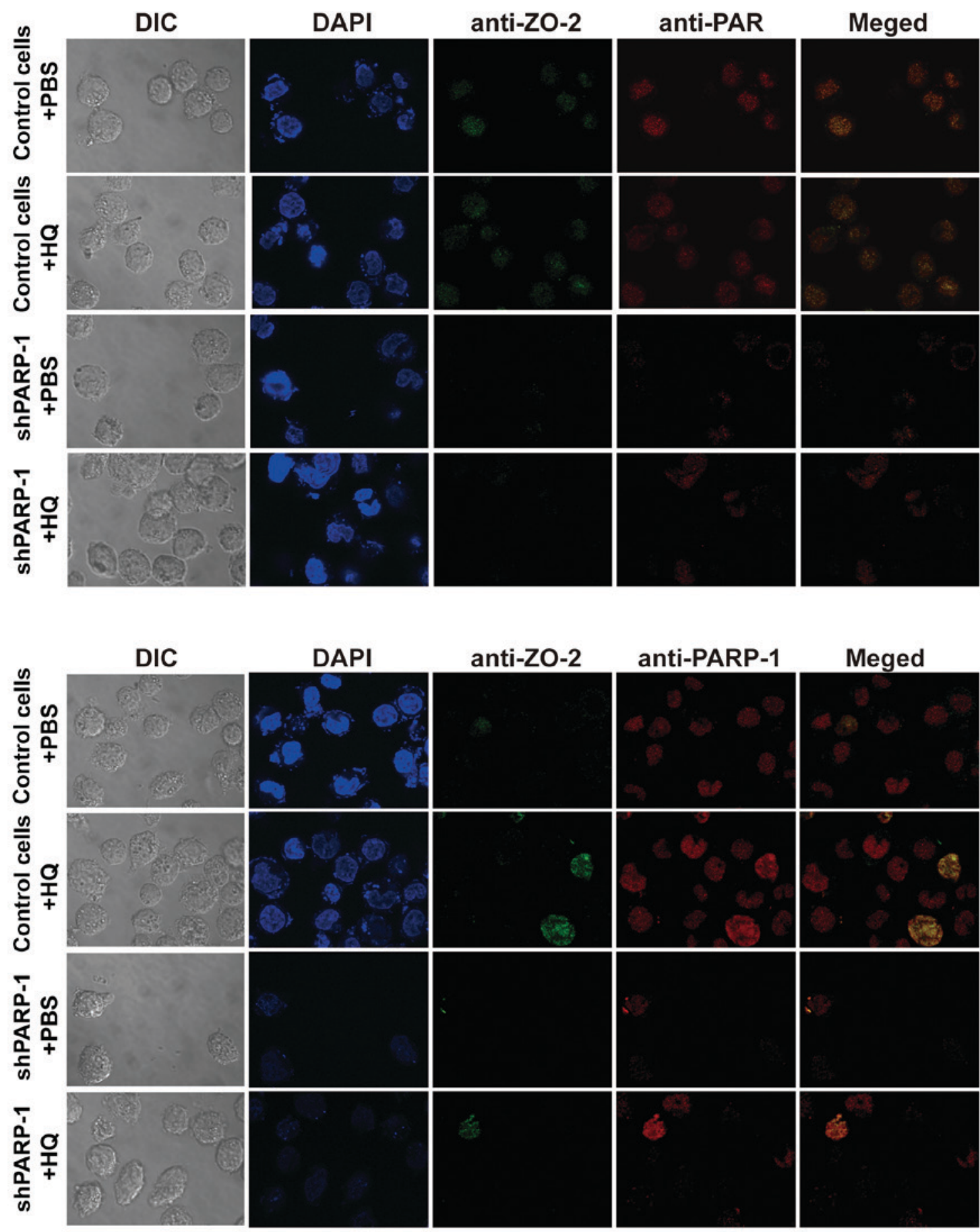

Figure 4. ZO-2 may be modified by PARylation. Cells were treated with $10 \mu \mathrm{M}$ HQ for $72 \mathrm{~h}$ and then stained using indirect immunofluorescence for ZO-2 (green), PAR (red, upper panel), PARP-1 (red, lower panel) and DAPI (blue) and the ZO-2 protein was modified by PARylation (upper panel) via an interaction with PARP-1 (lower panel). Magnification, x1,000. ZO-2, zona occludens 2; DIC, differential interference contrast; HQ, hydroquinone; PARP-1, poly (ADP-ribose) polymerase-1; shPARP-1, short hairpin PARP-1; PAR, poly (ADP-ribose).

HQ-induced apoptosis involved caspase-dependent intrinsic apoptosis.

The present study demonstrated that in TK6 cells, PARP-1 knockdown via RNA interference (RNAi) partially blocked apoptosis in cells exposed to a low dose of HQ for a prolonged time period. A recent study has also revealed that knockdown of PAPR-1 prevents apoptosis induced by HQ (28). Additionally, the present study determined that the mechanisms underlying this protection may involve the activation of Bcl-2 and the production of ATP and ROS. HQ-induced apoptosis was reduced from $16.66 \pm 0.47$ to $9.61 \pm 0.17 \%$ when PARP-1 was knocked down, indicating that PARP-1 regulates HQ-induced apoptosis. PARP-1 knockdown cells that were treated with HQ, Bcl-2 was upregulated and caspase-3 levels were not changed. It is of note, HQ-induced caspase-3/7 activity and ATP production were enhanced by PARP-1 knockdown. These findings suggest that prolonged exposure to a low dose of HQ did not block the production of energy and that inhibition of PARP-1 in TK6 cells maintained ATP levels. Inhibition of PARP-1 prevented the depletion of NAD ${ }^{+}$ 
and ATP and partially restored the ability of cells to perform DNA, RNA, and protein synthesis. Therefore, in TK6 cells, PARP-1 knockdown via RNAi partially blocked apoptosis in cells treated a low dose of HQ for a prolonged period of time and that the mechanisms underlying this protection may involve the activation of $\mathrm{Bcl}-2$ and the production of ATP. Furthermore, HQ treatment increased the production of ROS, which was consistent with the findings previously reported in ARPE-19 human retinal pigment epithelial cells, R-28 rat retinal neurosensory cells, human microvascular endothelial cells and MIO-M1 human retinal Müller cells (29-31). A previous study has revealed that may HQ induce apoptosis through a ROS pathway (29). In the present study, inhibition of PARP-1 attenuated the production of ROS that was induced by HQ treatment, indicating that inhibiting PARP-1 may attenuate HQ-induced apoptosis partly via the ROS pathway.

PARylation facilitates DNA repair and therefore cell survival in response to mild DNA damage, whereas more severe genotoxic stimuli activate the apoptotic pathway, and the most severe DNA damage may lead to the excessive activation of PARP and the depletion of the cellular NAD ${ }^{+}$and ATP stores. Depleting NAD ${ }^{+}$and ATP blocks apoptosis and leads to necrosis $(10,32)$. The present study determined that apoptosis was induced by prolonged exposure to a low dose of HQ and that this effect may be partially reversed by inhibition of PARP-1; however, it is difficult identify PARP-1 as a survival or a cytotoxic factor that contributes to cell survival, death or deterioration following exposure to different doses of HQ for different periods of time in specific cell types. Therefore, more experiments are required to determine the precise mechanisms by which HQ induces toxicity and to identify in which of these mechanisms PARP-1 is involved.

Activated PARP-1 and PARylation regulate various cellular processes, such as replication, transcription, DNA repair and ATP metabolism, and they mediate various cellular phenomena, such as proliferation, differentiation, senescence and cell death $(33,34)$. The presence of ZO-2 in the nucleus was determined more than a decade ago; however, its role in lymphoblastoid cells that lack tight junctions remains to be fully elucidated. In the nucleus, ZO-2 reportedly interacts with several proteins, including Jun, Fos, c-Myc and PKA (35-37). Co-localization between ZO-2 and PAR (or PARP-1 protein) was demonstrated by the present study using immunofluorescence confocal microscopy, indicating that ZO-2 is PARylated via an interaction with PARP-1. These findings were consistent with its protein expression detected using western blot analysis and the co-localization revealed that treatment with HQ led to the upregulation of ZO-2 in control cells and its downregulation in shPARP-1 cells. To the best of our knowledge, the present study is the first to determine that PARylation may modify the $\mathrm{ZO}-2$ protein and to reveal that $\mathrm{ZO}-2$ is not distributed in the nucleus in lymphoblastoid cells that lack tight junctions.

$\mathrm{ZO}-2$ induces the expression of various apoptosis-associated genes, including Bcl-2, interleukin-6, REL proto-oncogene, $\mathrm{NF}-\kappa \mathrm{B}$ subunit and translocator protein (38). It interacts with the transcriptional activator Yes kinase-associated protein 2 (YAP2) and enhances the nuclear localization and pro-apoptotic function of YAP (39). When PARP-1 expression was inhibited in the present study, ZO-2 was downregulated in parallel with changes observed in apoptosis in HQ-treated cells. Therefore, ZO-2 acted as a promoter of HQ-induced apoptosis and these data confirmed that ZO-2 may act as a tumor-suppressing protein.

In summary, the findings of the present study indicated that prolonged exposure to a low dose of HQ induced TK6 cells to undergo apoptosis and that inhibition of PARP-1 attenuated cellular apoptosis by activation of Bcl-2, inducing energy-saving mechanisms and reducing ROS. During this HQ-induced process, ZO-2 localized into the nucleus in lymphoblastoid cells and ZO-2 was modified by PARP-1 via PARylation. Therefore, when investigating the molecular mechanisms of HQ-induced toxicity and the contribution of HQ to benzene-induced leukemia, it is important to consider pleiotropic PAPR-1 and its inhibitors during clinical treatment.

\section{Acknowledgements}

The present study was supported by grants from the National Natural Science Foundation of China (grant no. 81202231 to Dr Linhua Liu; grant no. 81273116 to Professor Huanwen Tang; a key program 81430079 to Professor Wen Chen, and grant no. 81372962 to Professor Yongmei Xiao), the Science and Technology Program of the Guangdong Bureau of Science and Technology, China (grant no. 2013B021800069 to Dr Linhua Liu), the Guangdong Provincial Natural Science Foundation, China (grant no. S2013010015153 to Professor Huanwen Tang, grant no. 2014KQNCX102 to Dr Xiaoxuang Ling), the Key Project of Science and Technology Program of Dongguan Bureau of Science and Technology, China (grant no. 2012108101011 to Professor Huanwen Tang), the Science and Technology Program of Zhanjiang Bureau of Science and Technology, China (grant no. 2013B01082 to Professor Huanwen Tang), and the Science Foundation of Guangdong Medical University, China (grant no. M2013004 to Professor Huanwen Tang).

\section{References}

1. Elmore S: Apoptosis: A review of programmed cell death. Toxicol Pathol 35: 495-516, 2007.

2. Fulda S: Cross talk between cell death regulation and metabolism. Methods Enzymol 542: 81-90, 2014

3. Oka S, Kato $\mathrm{J}$ and Moss J: Identification and characterization of a mammalian 39-kDa poly(ADP-ribose) glycohydrolase. J Biol Chem 281: 705-713, 2006.

4. Davidovic L, Vodenicharov M, Affar EB and Poirier GG: Importance of poly(ADP-ribose) glycohydrolase in the control of poly(ADP-ribose) metabolism. Exp Cell Res 268: 7-13, 2001.

5. Gibson BA and Kraus WL: New insights into the molecular and cellular functions of poly(ADP-ribose) and PARPs. Nat Rev Mol Cell Biol 13: 411-424, 2012

6. Virag L, Robaszkiewicz A, Rodriguez-Vargas JM and Oliver FJ: Poly(ADP-ribose) signaling in cell death. Mol Aspects Med 34: 1153-1167, 2013.

7. Galluzzi L, Vitale I, Abrams JM, Alnemri ES, Baehrecke EH, Blagosklonny MV, Dawson TM, Dawson VL, El-Deiry WS, Fulda S, et al: Molecular definitions of cell death subroutines: Recommendations of the nomenclature committee on cell death 2012. Cell Death Differ 19: 107-120, 2012.

8. De Vos M, Schreiber V and Dantzer F: The diverse roles and clinical relevance of PARPs in DNA damage repair: Current state of the art. Biochem Pharmacol 84: 137-146, 2012.

9. Curtin NJ and Szabo C: Therapeutic applications of PARP inhibitors: Anticancer therapy and beyond. Mol Aspects Med 34: 1217-1256, 2013. 
10. Virág L and Szabó C: The therapeutic potential of poly(ADP-ribose) polymerase inhibitors. Pharmacol Rev 54: 375-429, 2002.

11. McHale CM, Zhang L and Smith MT: Current understanding of the mechanism of benzene-induced leukemia in humans Implications for risk assessment. Carcinogenesis 33: 240-252, 2012.

12. Slater ME, Linabery AM, Spector LG, Johnson KJ, Hilden JM, Heerema NA, Robison LL and Ross JA: Maternal exposure to household chemicals and risk of infant leukemia: A report from the children's oncology group. Cancer Causes Control 22: 1197-1204, 2011.

13. North M, Tandon VJ, Thomas R, Loguinov A, Gerlovina I, Hubbard AE, Zhang L, Smith MT and Vulpe CD: Genome-wide functional profiling reveals genes required for tolerance to benzene metabolites in yeast. PLoS One 6: e24205, 2011.

14. Gaskell M, McLuckie KI and Farmer PB: Genotoxicity of the benzene metabolites para-benzoquinone and hydroquinone. Chem Biol Interact 153-154: 267-270, 2005.

15. Smith MT: The mechanism of benzene-induced leukemia: A hypothesis and speculations on the causes of leukemia. Environ Health Perspect 104 (Suppl 6): S1219-S1225, 1996.

16. Liu L, Ling $X$, Tang $H$, Chen J, Wen $Q$ and Zou $F$ : Poly(ADP-ribosyl)ation enhances H-RAS protein stability and causes abnormal cell cycle progression in human TK6 lymphoblastoid cells treated with hydroquinone. Chem Biol Interact 238: $1-8,2015$

17. Carter-O'Connell I, Jin H, Morgan RK, David LL and Cohen MS Engineering the substrate specificity of ADP-ribosyltransferases for identifying direct protein targets. J Am Chem Soc 136 5201-5204, 2014

18. Gumbiner B, Lowenkopf $\mathrm{T}$ and Apatira D: Identification of a $160-\mathrm{kDa}$ polypeptide that binds to the tight junction protein ZO-1. Proc Natl Acad Sci USA 88: 3460-3464, 1991.

19. Traweger A, Toepfer S, Wagner RN, Zweimueller-Mayer J, Gehwolf R, Lehner C, Tempfer H, Krizbai I, Wilhelm I, Bauer HC and Bauer H: Beyond cell-cell adhesion: Emerging roles of the tight junction scaffold ZO-2. Tissue Barriers 1: e25039, 2013.

20. Walsh T, Pierce SB, Lenz DR, Brownstein Z, Dagan-Rosenfeld O Shahin H, Roeb W, McCarthy S, Nord AS, Gordon CR, et al: Genomic duplication and overexpression of TJP2/ZO-2 leads to altered expression of apoptosis genes in progressive nonsyndromic hearing loss DFNA51. Am J Hum Genet 87: 101-109, 2010.

21. Liu L, Ling X, Liang H, Gao Y, Yang H, Shao J and Tang H: Hypomethylation mediated by decreased DNMTs involves in the activation of proto-oncogene MPL in TK6 cells treated with hydroquinone. Toxicol Lett 209: 239-245, 2012.

22. Yang $\mathrm{H}$, Liang $\mathrm{H}$, Chen $\mathrm{J}$ and $\mathrm{Xu} \mathrm{Y}$ : Construction of PARP-1 gene silencing cell lines by lentiviral-mediated RNA interference technology. J Environ Health 31: 288-291, 377, 2014.

23. Livak KJ and Schmittgen TD: Analysis of relative gene expression data using real-time quantitative PCR and the 2(-Delta Delta C(T)) method. Methods 25: 402-408, 2001

24. Li Z, Wang C, Zhu J, Bai Y, Wang W, Zhou Y, Zhang S, Liu X, Zhou S, Huang W, et al: The possible role of liver kinase B1 in hydroquinone-induced toxicity of murine fetal liver and bone marrow hematopoietic stem cells. Environ Toxicol 31: 830-841, 2016.
25. Huang J, Zhao M, Li X, Ma L, Zhang J, Shi J, Li B, Fan W and Zhou Y: The cytotoxic effect of the benzene metabolite hydroquinone is mediated by the modulation of MDR1 expression via the NF- $\mathrm{BB}$ signaling pathway. Cell Physiol Biochem 37: 592-602, 2015.

26. Siew EL, Chan KM, Williams GT, Ross D and Inayat-Hussain SH: Protection of hydroquinone-induced apoptosis by downregulation of Fau is mediated by NQO1. Free Radic Biol Med 53: $1616-1624,2012$

27. Cosentino K and Garcia-Sáez AJ: Mitochondrial alterations in apoptosis. Chem Phys Lipids 181: 62-75, 2014.

28. Sha Y, Zhou W, Yang Z, Zhu X, Yang X, Li TD and Zhu D: Down-regulation of poly (ADP-ribose) polymerase 1 leads to change of hydroquinone cytotoxicity in TK6 cells. Toxicol Mech Methods 25: 467-477, 2015.

29. Ramirez C, Pham K, Franco MF, Chwa M, Limb A, Kuppermann BD and Kenney MC: Hydroquinone induces oxidative and mitochondrial damage to human retinal Müller cells (MIO-M1). Neurotoxicology 39: 102-108, 2013.

30. Sharma A, Neekhra A, Gramajo AL, Patil J, Chwa M, Kuppermann BD and Kenney MC: Effects of Benzo(e)Pyrene, a toxic component of cigarette smoke, on human retinal pigment epithelial cells in vitro. Invest Ophthalmol Vis Sci 49: 5111-5117, 2008.

31. Patil AJ, Gramajo AL, Sharma A, Chwa M, Seigel GM, Kuppermann BD and Kenney MC: Effects of benzo(e)pyrene on the retinal neurosensory cells and human microvascular endothelial cells in vitro. Curr Eye Res 34: 672-682, 2009.

32. Kraus WL: PARPs and ADP-ribosylation: 50 years ... and counting. Mol Cell 58: 902-910, 2015.

33. Ryu KW, Kim DS and Kraus WL: New facets in the regulation of gene expression by ADP-ribosylation and poly(ADP-ribose) polymerases. Chem Rev 115: 2453-2481, 2015.

34. Bürkle A and Virág L: Poly(ADP-ribose): PARadigms and PARadoxes. Mol Aspects Med 34: 1046-1065, 2013.

35. Hernandez S, Chavez Munguia B and Gonzalez-Mariscal L: ZO-2 silencing in epithelial cells perturbs the gate and fence function of tight junctions and leads to an atypical monolayer architecture. Exp Cell Res 313: 1533-1547, 2007.

36. Betanzos A, Huerta M, Lopez-Bayghen E, Azuara E, Amerena J and González-Mariscal L: The tight junction protein ZO-2 associates with Jun, Fos and C/EBP transcription factors in epithelial cells. Exp Cell Res 292: 51-66, 2004.

37. Avila-Flores A, Rendon-Huerta E, Moreno J, Islas S, Betanzos A, Robles-Flores $M$ and González-Mariscal L: Tight-junction protein zonula occludens 2 is a target of phosphorylation by protein kinase C. Biochem J 360: 295-304, 2001.

38. Gonzalez-Mariscal L, Bautista P, Lechuga S and Quiros $\mathrm{M}$ : $\mathrm{ZO}-2$, a tight junction scaffold protein involved in the regulation of cell proliferation and apoptosis. Ann N Y Acad Sci 1257: $133-141,2012$

39. Oka T, Remue E, Meerschaert K, Vanloo B, Boucherie C, Gfeller D, Bader GD, Sidhu SS, Vandekerckhove J, Gettemans J and Sudol M: Functional complexes between YAP2 and ZO-2 are PDZ domain-dependent, and regulate YAP2 nuclear localization and signalling. Biochem J 432: 461-472, 2010. 